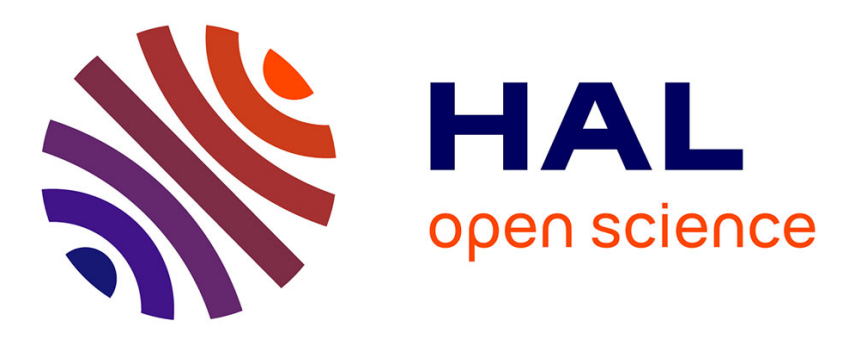

\title{
Bond anisotropy and cohesion of wet granular materials
}

Farhang Radjai, Vincent Richefeu

\section{To cite this version:}

Farhang Radjai, Vincent Richefeu. Bond anisotropy and cohesion of wet granular materials. Philosophical Transactions of the Royal Society of London. A (1887-1895), 2009, 367 (1909), pp.5123-5138. 10.1098/rsta.2009.0185. hal-00689863

\section{HAL Id: hal-00689863 https://hal.science/hal-00689863}

Submitted on 20 Apr 2012

HAL is a multi-disciplinary open access archive for the deposit and dissemination of scientific research documents, whether they are published or not. The documents may come from teaching and research institutions in France or abroad, or from public or private research centers.
L'archive ouverte pluridisciplinaire $\mathbf{H A L}$, est destinée au dépôt et à la diffusion de documents scientifiques de niveau recherche, publiés ou non, émanant des établissements d'enseignement et de recherche français ou étrangers, des laboratoires publics ou privés. 


\title{
Bond anisotropy and cohesion of wet granular materials
}

\author{
By Farhang Radjai* and Vincent Richefeu** \\ * LMGC, CNRS-Université Montpellier 2, Place Eugène Bataillon, 34095 \\ Montpellier, Cedex 5, France. \\ ${ }^{*} *$ L3S-R, Domaine Universitaire, B.P. 53, 38041 Grenoble, Cedex 9, France.
}

We analyze the Coulomb cohesion of wet granular materials and its relationship with the distribution of capillary bonds between the particles. We show that, within a harmonic representation of the bond and force states, the shear strength can be expressed as a state equation in terms of internal anisotropy parameters. This formulation involves a dependence of the shear strength on loading direction and leads to the fragile behavior of granular materials. The contact dynamics simulations of a wet material, in which a capillary force law is prescribed, are in excellent agreement with the predictions of this model. We find that the fragile character decreases as the local adhesion is increased or the mean stress is decreased.

\section{Keywords: granular matter, capillary bond, shear strength, Coulomb cohesion, jamming, fragile behavior}

\section{Introduction}

Wet granular materials in the pendular state are characterized by a network of liquid bonds inducing capillary attractive forces between neighboring particles. This network is equilibrated by repulsive contact forces and it endows the material with an overall capillary cohesion [8]. The capillary cohesion has been widely investigated for its crucial role in flow and mixing properties of granular materials. Wet processing is common in powder technology for operations such as granulation, extrusion and compaction $[2,7]$. In the same way, the cohesion of unsaturated soils is a fundamental parameter for construction environments such as embankments and excavations $[19,15,14]$.

The capillary force between two particles results from (1) the surface tension at the contact line between the liquid and the particles, and (2) the suction pressure difference due to the curvature of the liquid bridge. The pendular state represents both the simplest topology of the liquid phase and the highest level of capillary cohesion. The cohesion is absent at very low liquid content, and rises to an almost constant value as a function of liquid content for the liquid volume fraction in the range $1-3 \%$ [12]. This plateau cohesion has been evidenced for various materials and liquids $[24,37,32,21]$. At larger liquid contents, liquid clusters are formed in the packing with increasingly lower liquid-gas interfacial energy and hence lower overall cohesion [8].

An interesting issue is how the capillary cohesion depends on the bond force and granular microstructure. Assuming the Mohr-Coulomb model, the cohesion is given by the product of the tensile strength and the internal friction coefficient. 
The most widely cited expression of tensile strength was formulated by Rumpf [36]. This expression has often been found to be plausible in view of experimental measurements and numerical simulations [24, 10, 15]. It correctly predicts that the tensile strength varies in inverse proportion to the particle size and in proportion to the solid fraction and bond coordination number, which are the only structural parameters involved in this model. An expression of the Coulomb cohesion based on a variant of Rumpf's expression taking into account the polydispersity was also found in good agreement with numerical and experimental data [32]. However, the distribution of capillary bridge volumes and coordination numbers, involved in those expressions, have only recently been investigated by rigorous experimental methods as a function of liquid content $[16,8]$.

In this paper, we introduce a somewhat different picture of the cohesion of granular materials. The point is that the Coulomb cohesion is part of the plastic yield state of a granular material, and in this sense it is a function of the internal parameters pertaining to the granular microstructure [35]. In other words, the cohesion is a state-dependent property and a material should be characterized by its state of cohesion. In particular, it depends not only on the connectivity of the bond network, as a scalar parameter, but also on its anisotropy, which depends on the preparation process and evolves during shear. The internal angle of friction and cohesion are often attributed either to the stress peak state or to the critical state reached at large shear strains. Even at these particular states, the anisotropy of the bond network implies that the cohesion and internal friction angle are not isotropic properties but dependent on space direction [27]. For example, the cohesion changes as the shear strain is reversed, a property that is akin to the fragile behavior, defined as the resistance of a material only to a set of compatible stresses, basically those applied during its past deformations [5].

In the following, we first present a model of the capillary bond force in $\S 2$ and briefly discuss its properties. Since we are interested in the relationship between the Coulomb capillary cohesion and granular microstructure, we introduce in $\S 3$ a state equation for the cohesion of a granular material within the harmonic representation of the fabric and force states. In $\S 4$ we show that the predictions of this equation are in good agreement with contact dynamics simulations both for cohesive and cohesionless materials. This equation implies a fragile behavior that will be investigated as a function of the bond force. In $\S 5$ we derive an expression of the critical-state Coulomb cohesion as a function of the extra force and fabric anisotropies due to cohesion, and we show that it nicely fits the numerical data. We conclude with a summary and possible extensions of this work.

\section{Capillary cohesion}

\section{(a) Capillary bond force}

The capillary force $f_{n}^{c}$ between two spherical particles of radii $R_{i}$ and $R_{j}$ acts along the axis joining the particle centers. It is a function of the liquid surface tension $\gamma$, the gap $\delta_{n}$, the liquid bond volume $V_{b}$ and the particle-liquid-gas contact angle $\theta$; see figure 1(a). The capillary force can be obtained by integrating the Laplace-Young equation $[17,20,37]$. Three examples are shown in figure 1(b) for different values of the bond volume $V_{b}$ and size ratio $r=\max \left\{R_{i} / R_{j} ; R_{j} / R_{i}\right\}$. These 

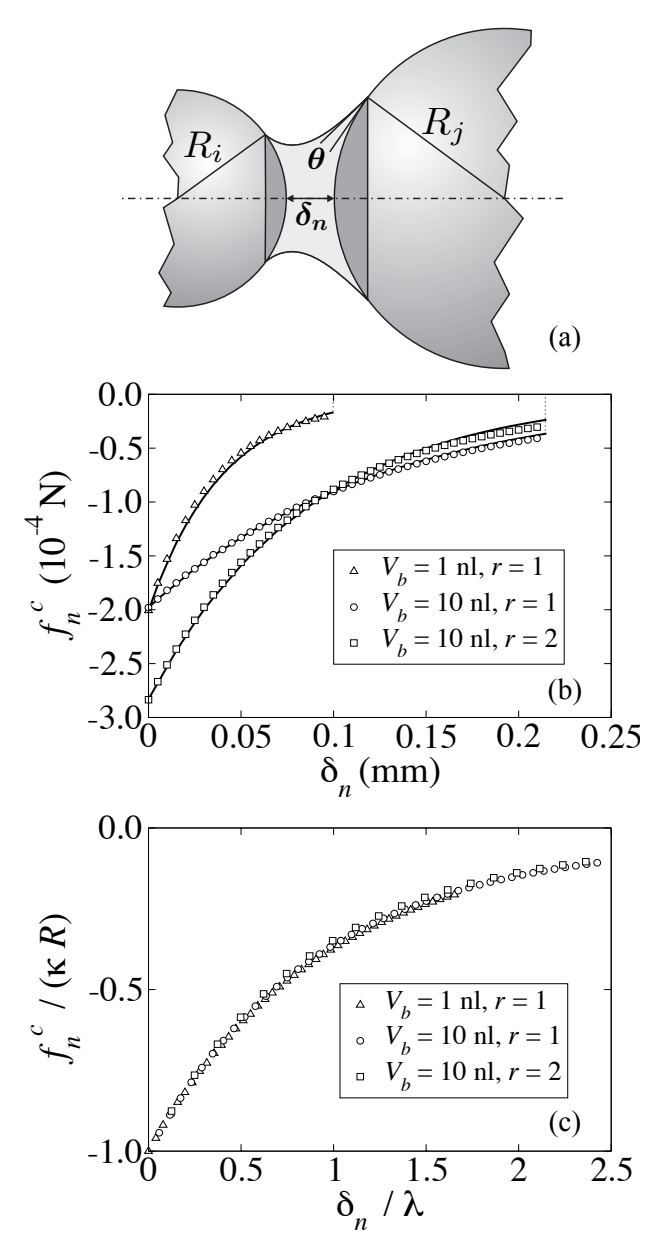

Figure 1. (a) Geometry of a capillary bridge; (b) Capillary force $f_{n}^{c}$ as a function of the gap $\delta_{n}$ between two particles for different values of the liquid volume $V_{b}$ and size ratio $r$ (solid lines), and from direct integration of the Laplace-Young equation (open circles); (c) Scaled plot of the capillary force as a function of gap from the direct data shown in (b).

data are nicely fit to an exponential form [31]:

$$
f_{n}^{c}=-\kappa R e^{-\delta_{n} / \lambda}
$$

where $R=\sqrt{R_{i} R_{j}}$ is the geometrical mean of particle radii, and $\lambda$ is a length scale characterizing the exponential fall-off (see below).

The parameter $\kappa$ in equation (2.1) is given by [39, 3]

$$
\kappa=2 \pi \gamma \cos \theta
$$

and $\delta_{n}^{\max }$ is the debonding distance given by [18]

$$
\delta_{n}^{\max }=\left(1+\frac{\theta}{2}\right) V_{b}^{1 / 3}
$$

The capillary bridge is stable for $\delta_{n}<\delta_{n}^{\max }$. The prefactor $\kappa R$ characterizes the highest value of the capillary force, occurring at contact $\left(\delta_{n}=0\right)$. 
The length $\lambda$ is expected to depend on the liquid volume $V_{b}$, a reduced radius $R^{\prime}$ and the ratio $r$. A dimensionally simple choice is

$$
\lambda=\alpha h(r)\left(\frac{V_{b}}{R^{\prime}}\right)^{1 / 2}
$$

where $\alpha$ is a constant prefactor, $h$ is a function of the ratio $r$, and $R^{\prime}$ is the harmonic mean $\left(R^{\prime}=2 R_{i} R_{j} /\left(R_{i}+R_{j}\right)\right)$. When introduced in equation (2.1), this form yields a nice fit to the capillary force obtained from direct integration of the Laplace-Young equation by setting $h(r)=r^{-1 / 2}$ and $\alpha \simeq 0.9$; see figure $1(\mathrm{~b})$. Figure $1(\mathrm{c})$ shows the same plots for forces normalized by $\kappa R$ and the lengths by $\lambda$. We see that all the data collapse on the same plot, indicating that the force $\kappa R$ and the expression of $\lambda$ in equation (2.4) describe correctly the capillary bond force. We checked that the geometric mean $R=\sqrt{R_{i} R_{j}}$ introduced in equation (2.1) provides a better fit than the harmonic mean $2 R_{i} R_{j} /\left(R_{i}+R_{j}\right)$ proposed by Derjaguin for polydisperse particles in the limit of small gaps [11].

The force law (2.1) was implemented in a molecular dynamics software and used to investigate the shear behavior and force distributions in 3D packings of spherical particles $[32,31]$. By homogeneously distributing the liquid among all eligible pairs of neighboring particles (within the debonding distance and including interparticle contacts) in a weakly polydisperse packing, it was found that $85 \%$ of capillary bonds occur at the true contact points, the other bonds being stretched and mostly carrying small tensile forces. This means that, the capillary bond force can be plausibly approximated by an adhesion force

$$
f_{a}=2 \pi \gamma \sqrt{R_{i} R_{j}} \cos \theta
$$

acting exclusively at the contact points between particles. It is also remarkable that $f_{a}$ does not depend on the bridge liquid volume so that increasing the liquid content in the pendular state affects mainly the proportion of liquid bonds in the bulk up to a maximum that slightly depends on the solid fraction. The fact that the distribution of liquid bonds is strongly coupled with the contact network explains the presence of a plateau state.

The capillary attraction forces induce a network of self-stresses with a bipolar structure that was evidenced by numerical simulations in the absence of external stresses [31]. When external (boundary or bulk) forces are applied, the mechanical effect of cohesive bonds depends on the relative importance of internal (tensile) and external (compressive) stresses [26, 9]. In other words, the mechanical behavior is expected to depend only on the ratio of $f_{a}$ to the reference compressive force $p d^{D-1}$ simply defined from the mean compressive stress $p$, the mean particle size $d$ and space dimension $D$. Thus, the relevant local parameter for a cohesive granular material, irrespective of the origin of the threshold adhesion force $f_{a}$, is

$$
\eta=\frac{f_{a}}{p d^{D-1}}
$$

We will refer below to this parameter as adhesion index. For millimeter-size grains at the free surface of a humid beach sand, the typical compressive force is the grain weight $m g$ and we have $\eta \simeq 5$. This is a large adhesion index that underlies the stability of sand castles. 


\section{(b) Coulomb cohesion}

The macroscopic cohesion $c$ is defined by the Mohr-Coulomb criterion, which is a linear relation between the normal stress $\sigma_{n}$ and the shear stress $\sigma_{t}$; see figure 2 . The slope is the internal friction coefficient $\mu=\tan \varphi$ and the Coulomb cohesion $c$ is the shear stress at zero normal force. Plastic deformation occurs when in a plane across the material the condition $\left|\sigma_{t}\right|=\mu\left|\sigma_{n}\right|+c$ is fulfilled. This condition can be formulated in terms of the stress invariants. Let $\sigma$ be the stress tensor, and $\sigma_{1}$ and $\sigma_{2}=\sigma_{3}$ the principal stresses under axial symmetry for simplicity. We have $p=\left(\sigma_{1}+2 \sigma_{2}\right) / 3$ and we set $q=\left(\sigma_{1}-\sigma_{2}\right) / 3$ as the single nonzero stress deviator due to axial symmetry. Then, it can easily be shown from the Mohr-Coulomb yield criterion that the relative stress deviator $q / p$ at yield is given by

$$
\frac{q}{p}=\frac{2}{3-\sin \varphi}\left(\sin \varphi+\frac{c}{p} \cos \varphi\right) \quad \text { in } 3 \mathrm{D}
$$

In $2 \mathrm{D}$, we have $q=\left(\sigma_{1}-\sigma_{2}\right) / 2$ and $p=\left(\sigma_{1}+\sigma_{2}\right) / 2$, and we get

$$
\frac{q}{p}=\sin \varphi+\frac{c}{p} \cos \varphi \quad \text { in } 2 \mathrm{D}
$$

As for the local adhesion, the state of cohesion in a granular material is not characterized by only the macroscopic cohesion $c$, but rather by the ratio $c / p$ which appears at the same level as $\sin \varphi$ is the expressions (2.7) and (2.8), and which is linked with the internal state parameters, as we shall see below. We will also see that the critical-state value of $c / p$ is a nonlinear function of $\eta$.

\section{Force and fabric states}

(a) Stress tensor and state parameters

In order to describe the state of cohesion, we need a representation of the internal states pertaining to the microstructure and force transmission in a granular material. The classical expression of the stress tensor $\sigma$ contains the necessary information. Let $\vec{f}^{\alpha}$ be the contact force at the contact $\alpha$ between two particles and $\vec{\ell}^{\alpha}$ the branch vector joining the particle centers. The stress tensor is given by $[34,4,23]$

$$
\sigma_{i j}=n_{b}\left\langle\ell_{i}^{\alpha} f_{j}^{\alpha}\right\rangle_{\alpha}
$$

where $n_{b}$ is the number density of the bonds and $\langle\ldots\rangle_{\alpha}$ designs averaging over all bonds inside a control volume. This expression shows clearly that the stress tensor is a function of state for a granular material when the internal state is represented by the set $\left\{\overrightarrow{f^{\alpha}}, \vec{\ell}^{\alpha}\right\}$.

In practice, however, we need a statistical description due to granular disorder. In a statistical approach, the internal state is represented by the joint probability density function $P_{\ell f}(\vec{\ell}, \vec{f})$ of bond forces and branch vectors, and the stress tensor can be expressed by an integral

$$
\sigma_{i j}=n_{b} \int_{\mathcal{A}_{\ell}} \int_{\mathcal{A}_{f}} \ell_{i} f_{j} P_{\ell f}(\vec{\ell}, \vec{f}) d \vec{\ell} d \vec{f}
$$

where $\mathcal{A}_{\ell}$ and $\mathcal{A}_{f}$ are the integration domains of $\vec{\ell}$ and $\vec{f}$, respectively. 
At this stage, it is convenient to consider the force components $f_{n}$ and $f_{t}$ in the local reference frame $(\vec{n}, \vec{t})$, where $\vec{n}$ is the unit vector along the branch vector such that $\vec{\ell}=\ell \vec{n}$, and $\vec{t}$ is an orthogonal unit vector. We have $\vec{f}=f_{n} \vec{n}+f_{t} \vec{t}$. We also define the functions $P(\vec{n}),\left\langle f_{n}\right\rangle(\vec{n}),\left\langle f_{t}\right\rangle(\vec{n})$ and $\langle\ell\rangle(\vec{n})$ by the following relations:

$$
\begin{aligned}
P(\vec{n}) & =\int_{\ell=0}^{\infty} \int_{\mathcal{A}_{f}} P_{\ell f}(\vec{\ell}, \vec{f}) d \ell d \vec{f} \\
\langle\ell\rangle(\vec{n}) P(\vec{n}) & =\int_{\mathcal{A}_{f}} \ell P_{\ell f}(\vec{\ell}, \vec{f}) d \vec{f} \\
\left\langle f_{n}\right\rangle(\vec{n}) P(\vec{n}) & =\int_{\ell=0}^{\infty} \int_{\mathcal{A}_{f}} f_{n} P_{\ell f}(\vec{\ell}, \vec{f}) d \ell d \vec{f} \\
\left\langle f_{t}\right\rangle(\vec{n}) P(\vec{n}) & =\int_{\ell=0}^{\infty} \int_{\mathcal{A}_{f}} f_{t} P_{\ell f}(\vec{\ell}, \vec{f}) d \ell d \vec{f}
\end{aligned}
$$

The function $P(\vec{n})$ is the probability density function of the branch vector orientations (coinciding with the contact normals in the case of spherical particles or disks). Integrating (3.2) with respect to the force and considering the definitions (3.3), we get

$$
\sigma_{i j}=n_{b} \int_{\Omega}\langle\ell\rangle(\vec{n}) P(\vec{n})\left\{\left\langle f_{n}\right\rangle(\vec{n}) n_{i}(\vec{n})+\left\langle f_{t}\right\rangle(\vec{n}) t_{j}(\vec{n})\right\} d \vec{n}
$$

where $\Omega$ is the angular domain of integration.

\section{(b) Harmonic approximation}

The information contained in the functions $P(\vec{n}),\left\langle f_{n}\right\rangle(\vec{n}),\left\langle f_{t}\right\rangle(\vec{n})$ and $\langle\ell\rangle(\vec{n})$ is still too rich to be tractable experimentally or theoretically. In general, however, as a result of granular disorder, steric exclusions and mechanical equilibrium, these functions can not take arbitrary form. It is usually observed that they can be approximated by low-order terms of spherical harmonics in 3D and Fourier series in $2 \mathrm{D}[34,4]$. To avoid unnecessary complication, we consider here a 2D packing of disks and expand these functions in Fourier series truncated beyond the second order as a function of the orientation $\theta$ of $\vec{n}$ :

$$
\begin{aligned}
P(\theta) & \simeq \frac{1}{2 \pi}\left\{1+a_{b} \cos 2\left(\theta-\theta_{b}\right)\right\} \\
\langle\ell\rangle(\theta) & \simeq \ell_{m}\left\{1+a_{\ell} \cos 2\left(\theta-\theta_{b}\right)\right\} \\
\left\langle f_{n}\right\rangle(\theta) & \simeq f_{m}\left\{1+a_{n} \cos 2\left(\theta-\theta_{f}\right)\right\} \\
\left\langle f_{t}\right\rangle(\theta) & \simeq f_{m} a_{t} \sin 2\left(\theta-\theta_{f}\right)
\end{aligned}
$$

In this approximation, the state is characterized by the average branch vector length $\ell_{m}$, the fabric or bond anisotropies $a_{b}$ and $a_{\ell}$, the bond privileged direction $\theta_{b}$, the average force $f_{m}$, the force anisotropies $a_{n}$ and $a_{t}$, and the force privileged direction $\theta_{f}$. We must add the coordination number $z$ or the bond number density $n_{b}$ which appears in the prefactor to (3.4). The sine function for the expansion of the orthoradial component $\left\langle f_{t}\right\rangle(\theta)$ is imposed by the requirement that the mean orthoradial force is zero, satisfying the balance of force moments on the particles $\left(\int P(\theta)\left\langle f_{t}\right\rangle(\theta) d \theta=0\right)$. We will refer to the above expansions and the corresponding state parameters as a harmonic approximation of the granular state.

It should be remarked that part of the information involved in the angular force distributions is redundant since for a mean stress state $\sigma$ the contact forces can be partially determined for the specified contact network by means of the force and moment balance conditions up to some degree of indeterminacy resulting from the 
assumption of perfect particle rigidity and Coulomb friction law [40]. However, the contact forces reflect subtle features of the granular microstructure that are more evident to observe through the force network. The surprising inhomogeneity of the force chains could hardly be guessed just from the appearance of the contact network. The inclusion of the forces in the state is therefore a genuine choice in view of taking advantage of the well-known properties of the force network. Owing to their connection with the microstructure, the forces represent the state of the microstructure and, in the last analysis, they can be considered as fabric parameters for a given material. On the other hand, a proper sampling of the forces in regular and irregular grain configurations suggests that the behavior of the statistical distribution of forces in the range of weak forces is correlated with shear-induced force anisotropy [40].

\section{(c) State equations and fragile behavior}

Inserting the Fourier expansions (3.5) in equation (3.4), and integrating with respect to $\theta$, we arrive at the following relations for the stress state:

$$
\begin{aligned}
p & \simeq \frac{1}{2} n_{b} \ell_{m} f_{m} \\
\frac{q}{p} \cos 2 \theta_{\sigma} & \simeq \frac{1}{2}\left\{\left(a_{b}+a_{\ell}\right) \cos 2 \theta_{b}+\left(a_{n}+a_{t}\right) \cos 2 \theta_{f}\right\}
\end{aligned}
$$

where $\theta_{\sigma}$ is the major principal stress direction and the cross products among the anisotropies have been neglected. The same relations hold also in 3D under axial symmetry with the factor $1 / 2$ replaced by $2 / 5$ [1]. The two relations (3.6) and (3.7) are state functions of a granular assembly in the thermodynamic sense in the framework of harmonic approximation.

Equation (3.7) reveals an important property of granular plasticity: The shear strength $q / p$ reflects the ability of a granular system to develop force and bond anisotropies. This aspect was first demonstrated many years ago by Rothenburg and Bathurst [34]. Except in transients, the fabric and force states are co-axial with the stress state so that $\theta_{b}=\theta_{f}=\theta_{\sigma}$. As a result, we have $q / p \simeq 0.5\left(a_{b}+a_{\ell}+a_{n}+a_{t}\right)$ during a monotonic deformation. The anisotropy $a_{\ell}$ of the branch vector lengths can be small but takes non-negligible values for polydisperse systems and nonspherical particles $[1,38]$. The relative values of the other anisotropies depend on the composition (shape and particle sizes). It is also important to remark that $q / p$ does not directly depend on the coordination number $z$, which reflects the compactness of the material.

Here, we would like to underline another important property resulting from the phase differences $\theta_{\sigma}-\theta_{b}$ and $\theta_{\sigma}-\theta_{f}$ in equation (3.7). Owing to the phase factors, the shear strength $q / p$ depends on the loading direction. For example, the shear stress is $q_{1} / p \simeq 0.5\left(a_{b}+a_{\ell}+a_{n}+a_{t}\right)$ for $\theta_{\sigma}=\theta_{f}=\theta_{b}$ and $q_{2} / p \simeq 0.5\left(-a_{b}-a_{\ell}+a_{n}+a_{t}\right)$ for $\theta_{\sigma}=\theta_{f}=\theta_{b}+\pi / 2$. This corresponds to a difference of strength of the order of $a_{b}+a_{\ell}$ between the two directions. As a result, it is expected that when the loading direction $\theta_{\sigma}$ is reversed (i.e. for a rotation of $\pi / 2$ of the applied stress directions), the phase factor $\cos 2 \theta_{\sigma}$ changes sign as well as $\cos 2 \theta_{f}$ which reacts immediately to the stress, but $\cos 2 \theta_{b}$ does not react instantly since the evolution of the bonds requires particle rearrangements. Therefore, a discontinuous loss of strength occurs 
during such transients. This property is akin to the fragile behavior [5]. Here, we have a clear formulation of this property, which can be formulated in a weaker form by stating that the largest strength occurs along the loading path that conducted the system to its present state. In the following, we illustrate these developments by means of discrete element simulations.

\section{Numerical simulations}

(a) Contact dynamics method

For the simulations, we used the contact dynamics (CD) method. This method is based on implicit time integration and nonsmooth formulation of mutual exclusion and dry friction between particles [13, 22, 25, 6, 30]. The equations of motion are formulated as differential inclusions in which velocity jumps replace the accelerations. The unilateral contact interactions and Coulomb friction law are represented as set-valued force laws. The implementation of the time-stepping scheme requires the geometrical description of each potential contact in terms of contact position and its normal unit vector.

At each time step, all kinematic constraints implied by enduring contacts are simultaneously taken into account together with the equations of motion in order to determine all velocities and contact forces in the system. This problem is solved by an iterative process pertaining to the non-linear Gauss-Seidel method that consists of solving a single contact problem, with other contact forces being treated as known, and iteratively updating the forces until a given convergence criterion is achieved. The method is thus able to deal properly with the nonlocal character of the momentum transfers resulting from the impenetrability of the rigid particles and friction law.

The CD method is unconditionally stable due to its inherent implicit time integration method. The uniqueness of the solution at each time step is not guaranteed for perfectly rigid particles. However, by initializing each step with the forces calculated in the preceding step, the variability of admissible solutions shrinks to the numerical resolution.

In the simulations presented in this paper, the bond capillary force was taken into account only at the contact points between the particles as an attractive force given by equation (2.5) added to each contact. The total force at each contact results from the procedure briefly presented above in the presence of the attractive bond forces as well as other bulk and boundary forces acting on the system. As stated before, our 3D simulations with the full capillary law and an even distribution of the liquid bonds within the debonding distance indicate that the effect of stretched bonds (gap bridges) is marginal [32].

\section{(b) Samples and material parameters}

The numerical samples are composed of 5000 disks of diameters in a range $\left[d_{\min }, d_{\max }\right]$ with $d_{\max }=2 d_{\min }$. The samples are isotropically compacted with with friction and at zero gravity inside a rectangular box in which the bottom and left walls are immobile. We get an isotropic static sample of nearly square shape and solid fraction $\simeq 0.84$ when the whole energy is dissipated by inelastic collisions between the particles. In the CD method, the particles are perfectly rigid and the 
only material parameters are the normal and tangential restitution coefficients, set to zero in all simulations, and the coefficient of friction between the particles, set to 0.5 at the beginning of shearing.

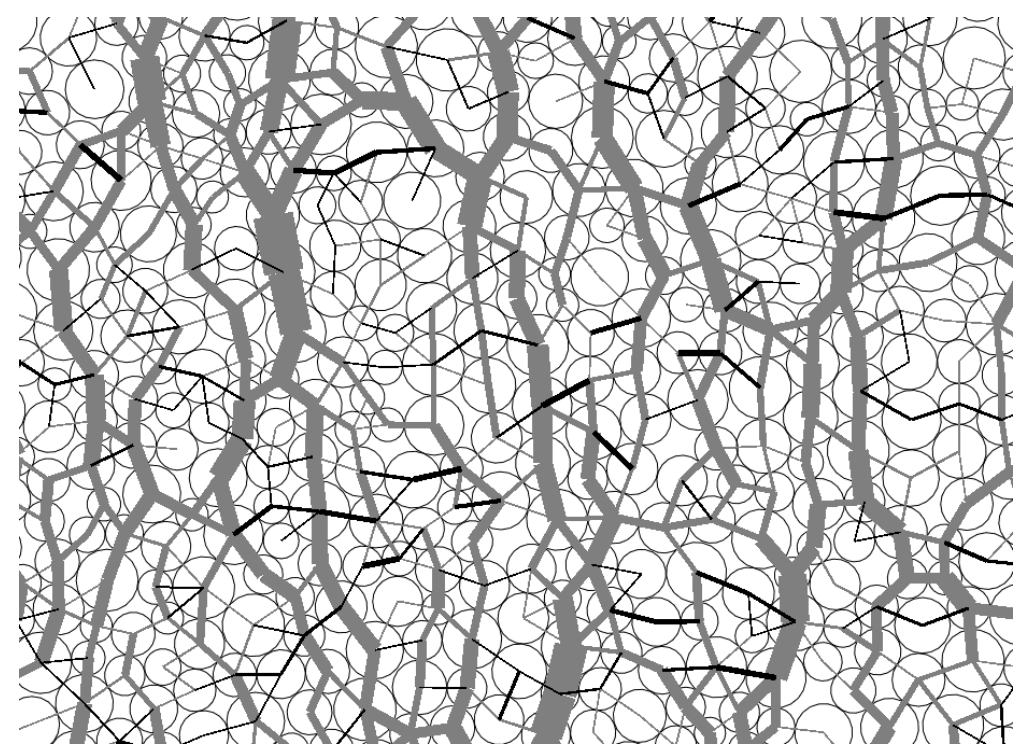

Figure 2. A snapshot of the force network in a portion of a cohesive sample. Line thickness is proportional to the magnitude of the normal force. The tensile and compressive forces are in black and grey, respectively.

The samples were sheared by the downward motion of the top wall at constant velocity $v_{y}$ and a constant confining pressure $\sigma_{x x}$ applied on the right wall. The vertical strain rate was $\dot{\varepsilon}_{y y} \simeq 0.02 \mathrm{~s}^{-1}$ and the corresponding inertial number $I \equiv \dot{\varepsilon}_{y y} \sqrt{m /\left(\sigma_{x x} d\right)} \simeq 10^{-4}$. This is weak enough to consider the deformation as quasistatic. The samples were sheared up to a total cumulative shear strain $\varepsilon_{q} \equiv$ $\int\left(\dot{\varepsilon}_{y y}-\dot{\varepsilon}_{x x}\right) d t=0.28$. Then, the simulation was stopped and a new simulation was started by reversing the direction of motion of the top wall. This reverse shearing was continued slightly below $\varepsilon_{q}=0$.

The samples differed only in the value of the adhesion index $\eta$. We present below the simulation results for six samples with $\eta$ varying in the range $[0,4]$.

\section{(c) Numerical results}

Figure 2 shows a snapshot of the bond forces in a portion of a sheared cohesive sample with $\eta \simeq 1$.4. Only normal bond forces are represented by line thickness and two grey levels differentiating the tensile and compressive forces. We observe both compressive and tensile force chains although the compressive forces prevail as the sample supports compressive stresses in both space directions.

The normalized stress deviator $(q / p) \cos 2 \theta_{\sigma}$ is displayed as a function of the cumulative shear strain $\varepsilon_{q}$ in figure 3 for a cohesionless and a cohesive sample, together with the corresponding fits by the state equation (3.7). The agreement is excellent all along the shear path including the transient after the shear strain 


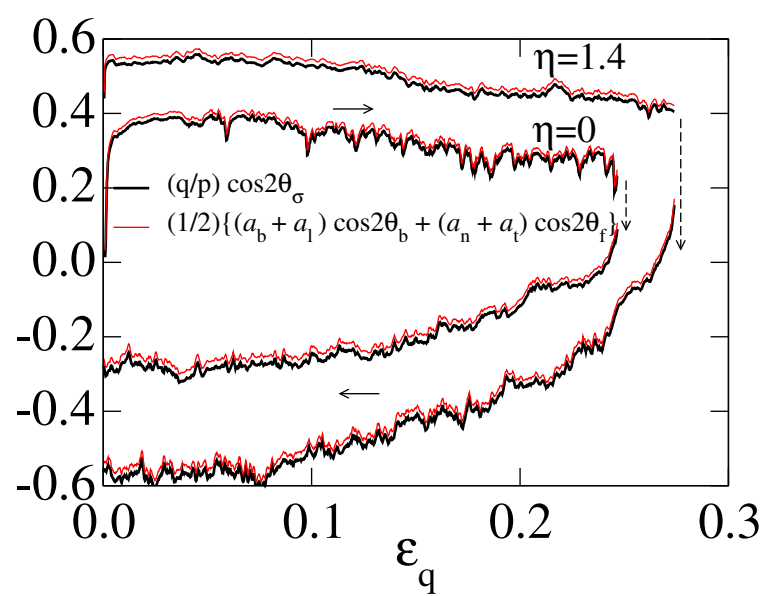

Figure 3. Normalized stress deviator $(q / p) \cos 2 \theta_{\sigma}$ as a function of the cumulative shear strain $\varepsilon_{q}$ together with the plot of the expression (3.7). The latter has been slightly translated upward for a better visibility of both plots.

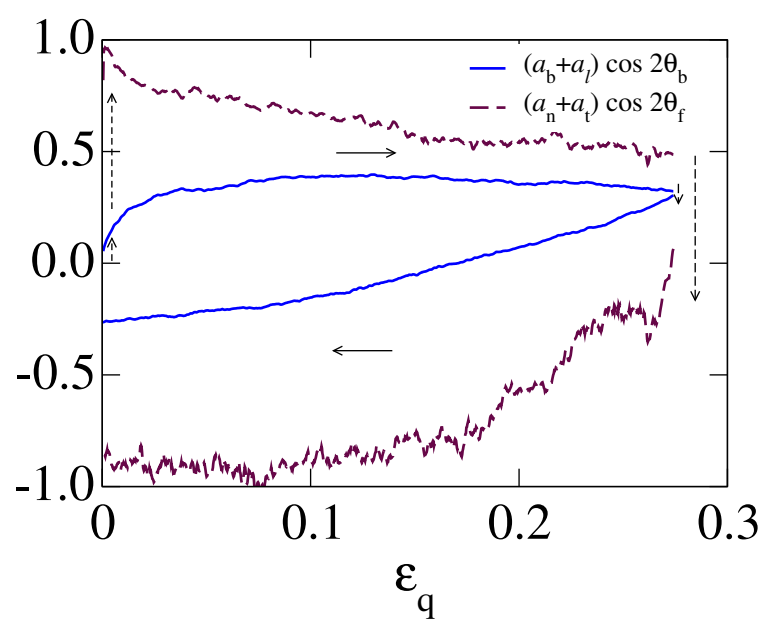

Figure 4. Evolution of the total fabric anisotropy $a_{b}+a_{\ell}$ and the total force anisotropy $a_{n}+a_{t}$ in the case of the cohesive packing of figure 3 (for $\eta=1.4$ ).

reversal. Starting with an initially isotropic system, the stress deviator increases almost monotonically (ignoring small scale fluctuations) with shear strain. In the case of perfectly rigid particles, which is the case of our simulations, this increase in shear resistance is a purely hardening effect. In other words, the initial elastic regime generally observed in simulations with elastic contacts (by means of other distinct element methods of "molecular dynamics" type) is totally absent from our results. Since the packing is initially dense, the stress ratio reaches a peak before declining to its critical state value (shear softening). Instead, in our system the stress deviator undergoes a huge jump over the first time step. This is the reminiscence of a rigid-plastic behavior. However, particle rearrangements take over afterwards and the behavior is then governed by the evolution of the microstructure. A similar jump occurs also at the moment of shear reversal but the particle rearrangements 
are again responsible for the long transient towards the critical state in the new stress direction.

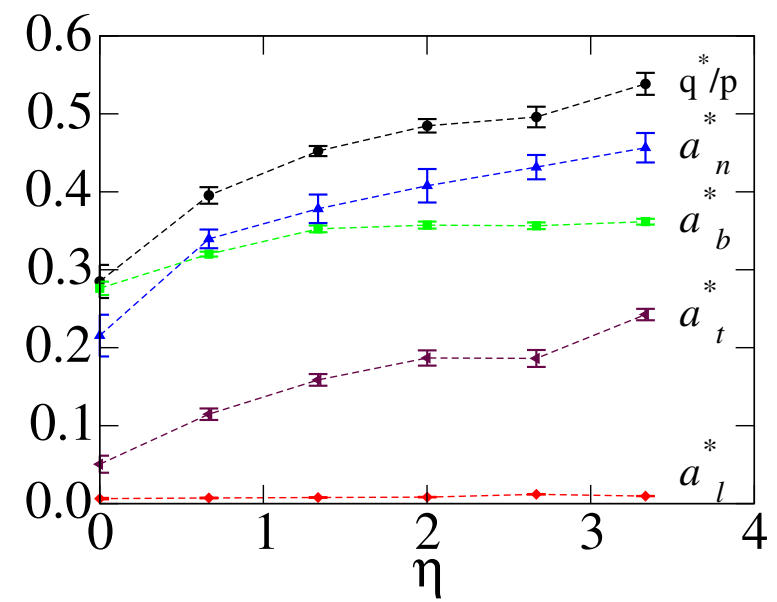

Figure 5. Critical-state values of the stress deviator and anisotropy parameters as a function of the adhesion index. The data are mean values in the critical state. The error bars represent the standard deviation of the fluctuations around the mean.

The stress-strain behavior is basically similar in both cohesive and cohesionless packings. The stress deviator is larger in the cohesive packing due to the action of tensile bonds. The fragile behavior is apparent at shear reversal where the stress deviator almost vanishes. As discussed previously, this is mainly due to the responsive nature of bond forces. The evolution of the fabric and force anisotropies is shown in figure 4 for the cohesive packing of figure 3 . We observe the slow evolution of the fabric anisotropy $a_{b}+a_{\ell}$ both at the initial state and upon strain reversal where a long transient occurs. In contrast, the force anisotropy $a_{n}+a_{t}$ undergoes a jump in both cases. This shows that the stress peak occurring in an initially dense packing is a consequence of the spontaneous buildup of force anisotropy in response to the applied stress. The degree of fragility is related to the stress jump at strain reversal. If $(q / p) \cos 2 \theta_{\sigma}$ simply changes sign in response to strain reversal while keeping the same amplitude, the packing is not fragile as it resists to shear in the new direction with the same strength as in the initial direction. In all other cases there is some degree of fragility.

The state equation (3.7) suggests that the fragile character should increase as $a_{b}+a_{\ell}$ decreases. The critical-state stress deviator $q^{*} / p$ and the critical-state anisotropies $a_{b}^{*}, a_{\ell}^{*}, a_{n}^{*}$ and $a_{t}^{*}$ are shown in figure 5 as a function of the adhesion index $\eta$. In our system, $a_{\ell}^{*}$ is nearly zero and $a_{b}^{*}$ increases and saturates to a constant value as a function of $\eta$. Hence, the fragile character of our packings decrease slightly as the cohesion increases. In contrast, the force anisotropies increase significantly with $\eta$, and they are thus the main origin of the shear strength in a cohesive granular material. 


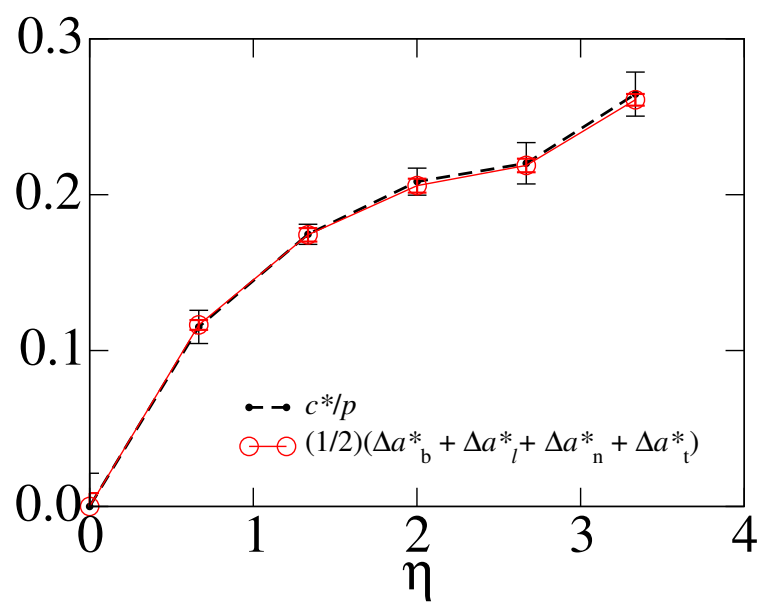

Figure 6. Critical-state cohesion $c^{*}$ and its theoretical expression (5.3) as a function of the adhesion index $\eta$. The error bars correspond to fluctuations around the mean in the critical state.

\section{Coulomb cohesion in the critical state}

The Coulomb cohesion $c$ of a packing can be obtained from equation (2.8) at any stage of evolution of a granular material if the corresponding internal friction angle $\varphi$ is known. In particular, the critical-state cohesion $c^{*}$ of a cohesive material of cohesion index $\eta$ in $2 \mathrm{D}$ is given by

$$
\frac{c^{*}(\eta)}{p}=\frac{1}{\cos \varphi^{*}}\left(\frac{q^{*}(\eta)}{p}-\sin \varphi^{*}\right)
$$

But $\varphi^{*}$ does not depend on the adhesion index and it represents the shear strength in the absence of adhesion, i.e. for $\eta=0$. Assuming that the phase differences vanish in the critical state $\left(\theta_{\sigma}=\theta_{b}=\theta_{f}\right)$, we have

$$
\sin \varphi^{*}=\frac{1}{2}\left\{a_{b}^{*}(0)+a_{\ell}^{*}(0)+a_{n}^{*}(0)+a_{t}^{*}(0)\right\}
$$

where the argument refers to the value of $\eta$. In the same way, under the same assumption, we have

$$
\frac{q^{*}(\eta)}{p}=\frac{1}{2}\left\{a_{b}^{*}(\eta)+a_{\ell}^{*}(\eta)+a_{n}^{*}(\eta)+a_{t}^{*}(\eta)\right\}
$$

Given the expression (5.2), $\cos \varphi^{*}$ is of second order with respect to the anisotropies. But in deriving (5.2) the second order terms (cross products among the anisotropies) were neglected. Hence, within this approximation, we set $\cos \varphi^{*} \simeq 1$. As a result, from (5.1), (5.2) and (5.3) we get the following expression for the critical-state Coulomb cohesion:

$$
\frac{c^{*}(\eta)}{p}=\frac{1}{2}\left\{\Delta a_{b}^{*}(\eta)+\Delta a_{\ell}^{*}(\eta)+\Delta a_{n}^{*}(\eta)+\Delta a_{t}^{*}(\eta)\right\}
$$

where $\Delta a_{b}^{*}(\eta)=a_{b}^{*}(\eta)-a_{b}^{*}(0), \Delta a_{\ell}^{*}(\eta)=a_{\ell}^{*}(\eta)-a_{\ell}^{*}(0), \Delta a_{n}^{*}(\eta)=a_{n}^{*}(\eta)-a_{n}^{*}(0)$, $\Delta a_{t}^{*}(\eta)=a_{t}^{*}(\eta)-a_{t}^{*}(0)$. This equation is in excellent agreement with our numerical 
simulations as displayed in figure 6. The four terms in equation (5.4) represent the contribution of adhesion to the structural and force anisotropy. Since $c^{*}$ is independent of $p$, this equation implies that these extra anisotropies tend to zero when the mean stress $p$ increases. From figure 5 we also see that $\Delta a_{n}^{*}(\eta) \simeq \Delta a_{t}^{*}(\eta)$, and $\Delta a_{b}^{*}(\eta)$ is small and nearly constant beyond $\eta \simeq 1$.

For a better understanding of the effect of adhesion, a particle-scale interpretation of the behavior of critical-state anisotropies is necessary. Schematically, the Coulomb cohesion results equally from two different mechanisms: (1) the stabilizing effect of the tensile bonds, and (2) the enhanced friction at the compressive contacts. The parameter $\Delta a_{n}^{*}$ reflects the importance of force chains. In a dry cohesionless packing, these chains are propped by the weak compressive forces [29, 28]. The tensile bonds play a similar role with respect to the force chains in the presence of cohesion $[26,33]$. On the other hand, the parameter $\Delta a_{t}^{*}$ is basically an effect of enhanced friction due to cohesion. Its increase with the cohesion index, in the same proportion as $\Delta a_{n}^{*}$, shows clearly this correlation.

\section{Conclusion}

The Coulomb cohesion of wet granular materials was analysed in this paper in terms of the force and fabric anisotropies. It was argued that these anisotropies are state parameters upon which depend the stress tensor. An expression of the shear stress was derived in this framework for a harmonic representation of the states. This expression was shown to be in excellent agreement with contact dynamics simulations of biaxial compression tests both in monotonous deformation and during transients for several values of the local adhesion. We showed that the fragile behavior, defined as the space-direction dependence of strength, is a consequence of the fabric anisotropy and its effect increases with cohesion. We also derived an expression for the critical-state cohesion, which is nicely fitted by the numerical data. The evolution of the fabric and force anisotropies with the adhesion between particles suggests that the tensile bonds and enhanced friction at compressive contacts are equally at the origin of the Coulomb cohesion. However, more extensive numerical investigation are required at this stage in order to fully validate this approach in extreme situations such as tensile loading at negative confining stresses.

The framework presented in this paper provides a generic methodology for the analysis of shear strength in granular materials. The influence of various material parameters such as particle shape and size as well as particle interactions can thus be described by considering separately each anisotropy parameter. Each parameter affects differently the force and fabric anisotropies and thus the shear strength. In particular, an upper bound can be obtained for the shear strength from the variability of each anisotropy parameter.

\section{References}

[1] E. Azéma, G. Saussine, and F. Radjai. Quasistatic rheology, force transmission and fabric properties of a packing of irregular polyhedral particles. Mechanics of Materials, to appear., 2009. 
[2] D.G. Bika, M. Gentzler, and J.N. Michaels. Mechanical properties of agglomerates. Powder Technology, 117:98-112, 2001.

[3] Lyderic Bocquet, Elisabeth Charlaix, and Frederic Restagno. Physics of humid granular media. Comptes Rendus Physique, 3(2):207-215, 2002.

[4] B. Cambou. From global to local variables in granular materials. In C. Thornton, editor, Powders and Grains 93, pages 73-86, Amsterdam, 1993. A. A. Balkema.

[5] M. E. Cates, J. P. Wittmer, J.-P. Bouchaud, and P. Claudin. Jamming, force chains, and fragile matter. Phys. Rev. Lett., 81(9):1841-1844, 1998.

[6] F. Dubois and M. Jean. Lmgc90 une plateforme de dveloppement ddie la modlisation des problmes d'intraction. In Actes du sixième colloque national en calcul des structures - CSMA-AFM-LMS -, volume 1, pages 111-118, 2003.

[7] S. Forrest, J. Bridgwater, P. R. Mort, J. Litster, and D. J. Parker. Flow patterns in granulating systems. Powder Technology, 30:91-96, 2002.

[8] Z. Fournier, D. Gerimichalos, S. Herminghaus, M. M. Kohonen, F. Mugele, M. Scheel, M. Schulz, B. Schulz, C. Schier, R. Seemann, and A. Shudelny. Mechanical properties of wet granular materials. Applied Physics: Condensed Matter, 17:477-502, 2005.

[9] F. A. Gilabert, J-N. Roux, and A. Castellanos. Computer simulation of model cohesive powders: influence of assembling procedure and contact laws on low consolidation states. Phys. Rev. E, 75(1 Pt 1):011303, Jan 2007.

[10] T. Gröger, U. Tüzün, and D.M. Heyes. Modelling and measuring of cohesion in wet granular materials. Powder Technology, 133:203-215, 2003.

[11] J. N. Israelachvili. Intermolecular and surface forces. Academic Press, London, 1993.

[12] S.M. Iveson, J.A. Beathe, and N.W. Page. The dynamic strength of partially saturated powder compacts: the effect of liquid properties. Powder Technology, 127:149-161, 2002.

[13] M. Jean and J. J. Moreau. Unilaterality and dry friction in the dynamics of rigid body collections. In Proceedings of Contact Mechanics International Symposium, pages 31-48, Lausanne, Switzerland, 1992. Presses Polytechniques et Universitaires Romandes.

[14] M. J. Jiang, S. Leroueil, and J. M. Konrad. Insight into shear strength functions of unsaturated granulates by DEM analyses. Computers and Geotechnics, 31:473-489, 2004.

[15] T.H. Kim and C. Hwang. Modeling of tensile strength on moist granular earth material at low water content. Engineering Geology, 69:233-244, 2003.

[16] M.M. Kohonen, D. Geromichalos, M. Scheel, C. Schier, and S. Herminghaus. On capillary bridges in wet granular materials. Physica A, 339:7-15, 2004. 
[17] G. Lian, C. Thornton, and M. Adams. A theoretical study of the liquid bridge forces between two rigid spherical bodies. Journal of Colloid and Interface Science, 161:138-147, 1993.

[18] G. Lian, C. Thornton, and M. J. Adams. Discrete particle simulation of agglomerate impact coalescence. Chemical Engineering Science, 53(19):33813391, 1998.

[19] S. H. Liu, D. A. Sun, and Y. Wang. Numerical study of soil collapse behavior by discrete element modelling. Computers and Geotechnics, 30:399-408, 2003.

[20] T. Mikami, H. Kamiya, and M. Horio. Numerical simulation of cohesive powder behavior in fluidized bed. Chemical Engineering Science, 53(10):1927-1940, 1998.

[21] P. C. F. Moller, and D. Bonn. The shear modulus of wet granular matter. EPL, 80:38002, 2007.

[22] J.J. Moreau. Some numerical methods in multibody dynamics: Application to granular materials. European Journal of Mechanics A/Solids, supp.(4):93-114, 1994. Formulation mathematiques tire du livre Contacts mechanics.

[23] H. Ouadfel and L. Rothenburg. Stress-force-fabric relationship for assemblies of ellipsoids. Mechanics of Materials, 33(4):201-221, 2001.

[24] P. Pierrat and H.S. Caram. Tensile strength of wet granular materials. Powder Technology, 91:83-93, 1997.

[25] F. Radjai. Multicontact dynamics of granular systems. Computer Physics Communications, 121-122:294-298, 1999.

[26] F. Radjai, I. Preechawuttipong, and R. Peyroux. Cohesive granular texture. In P.A. Vermeer, S. Diebels, W. Ehlers, H.J. Herrmann, S. Luding, and E. Ramm, editors, Continuous and discontinuous modelling of cohesive frictional materials, pages 148-159, Berlin, 2001. Springer Verlag.

[27] F. Radjai, H. Troadec, and S. Roux. Key features of granular plasticity. In S.J. Antony, W. Hoyle, and Y. Ding, editors, Granular Materials: Fundamentals and Applications, pages 157-184, Cambridge, 2004. RS.C.

[28] F. Radjai and D. E. Wolf. The origin of static pressure in dense granular media. Granular Matter, 1:3-8, 1998.

[29] F. Radjai, D. E. Wolf, M. Jean, and J.J. Moreau. Bimodal character of stress transmission in granular packings. Phys. Rev. Letter, 80:61-64, 1998.

[30] Farhang Radjai and Vincent Richefeu. Contact dynamics as a discrete element method. Mechanics of Materials, 2009.

[31] V. Richefeu, F. Radjai, and M. S. El Youssoufi. Stress transmission in wet granular materials. Eur. Phys. J. E, 21:359-369, Feb 2007. 
[32] Vincent Richefeu, Moulay Saïd El Youssoufi, and Farhang Radjai. Shear strength properties of wet granular materials. Phys. Rev. E, 73(5 Pt 1):051304, May 2006.

[33] Vincent Richefeu, Moulay Said El Youssoufi, Emilien Azéma, and Farhang Radjai. Force transmission in dry and wet granular media. Powder Technology, 190:258-263, 2009.

[34] L. Rothenburg and R. J. Bathurst. Analytical study of induced anisotropy in idealized granular materials. Geotechnique, 39:601-614, 1989.

[35] S. Roux and F. Radjai. Statistical approach to the mechanical behavior of granular media. In H. Aref and J.W. Philips, editors, Mechanics for a New Millennium, pages 181-196, Netherlands, 2001. Kluwer Acad. Pub.

[36] H. Rumpf. Zur theorie der zugfestigkeit von agglomeraten bei kraftübertragung an kontaktpunten. Chemical Engineering Technology, 42:538-540, 1970.

[37] F. Soulié, M. S. El Youssoufi, F. Cherblanc, and C. Saix. Capillary cohesion and mechanical strength of polydisperse granular materials. Eur. Phys. J. E, 21(4):349-357, Dec 2006.

[38] C. Voivret. Modélisation numérique des matériaux granulaires polydisperses. PhD thesis, Université Montpellier 2, 2008.

[39] C. Willett, M. Adans, S. Johnson, and J. Seville. Capillary bridges between two spherical bodies. Langmuir, 16:9396-9405, 2000.

[40] J. H. Snoeijer, T. J. H. Vlugt, M. van Hecke, and W. van Saarloos. Force Network Ensemble: A New Approach to Static Granular Matter. Phys. Rev. Lett., $92(5): 054302-1$, Feb 2004. 